\title{
Successes and challenges of PARP inhibitors in cancer therapy
}

\author{
Tiffany K. Ricks*, Haw-Jyh Chiu, Gwynn Ison, Geoffrey Kim, Amy E. McKee, Paul Kluetz \\ and Richard Pazdur
}

Office of Hematology and Oncology Products (OHOP), Office of New Drugs (OND), Center for Drug Evaluation and Research (CDER), U.S. Food and Drug Administration, Silver Spring, MD, USA

Keywords: PARP inhibitor, cancer, drug resistance, BRCA1, BRCA2, homologous recombination

\section{INTRODUCTION}

Poly (ADP-ribose) polymerases (PARPs) are a family of enzymes involved in cellular homeostasis, including DNA transcription, cell-cycle regulation, and DNA repair $(1,2)$. PARPs can detect DNA damage and bind to DNA single strand breaks (SSBs) through their $\mathrm{N}$-terminal zinc finger domains. DNA binding activates the C-terminal catalytic domain, which hydrolyzes $\mathrm{NAD}^{+}$to attach poly ADP-ribose (PAR) polymers covalently to nuclear proteins, including PARP itself. Negatively charged PAR polymers promote recruitment of DNA repair proteins, and auto-PARylation causes dissociation of PARPs from DNA, allowing completion of DNA repair. In the absence of PARP activity, unrepaired SSBs can lead to more deleterious double strand breaks (DSBs), which require high fidelity, homologous recombination (HR) or low fidelity, non-homologous end joining (NHEJ) for repair.

In vitro and in vivo studies have demonstrated that tumor cells harboring defects in DNA repair are highly sensitive to PARP inhibitors, leading to genomic instability and cell death. Two publications demonstrated the concept of synthetic lethality in BRCA-deficient cells treated with PARP inhibitors $(3,4)$. Cells lacking functional alleles of $B R C A$ are defective in HR repair and have an increased susceptibility to cause tumor development. Loss of BRCA or inhibition of PARP alone has little effect on in vitro and in vivo tumor growth; however, loss of function of both proteins enhances anti-tumor activity. Restoring BRCA expression blocks the cytotoxic effects of PARP inhibitor treatment.

Several clinical PARP inhibitors are under investigation in Phase 2 and Phase 3 clinical trials as monotherapy in cancers with DNA repair defects or in combination with radiation, chemotherapy, or other targeted agents (Table 1). Progress in PARP inhibitor development has led to the recent accelerated approval of Lynparza (olaparib) by the U.S. Food and Drug Administration (5). Lynparza is currently indicated as monotherapy for patients with advanced germline $B R C A$-mutated ovarian cancer who have received three or more prior lines of chemotherapy. Lynparza was approved with a companion diagnostic test to select patients with deleterious or suspected deleterious $B R C A$ mutations. PARP inhibitors are anticipated to have a much broader clinical application in additional tumor types, particularly those with DNA repair defects and in combination with chemotherapy and other targeted agents. In light of renewed interest in PARP inhibitors and the recent approval of Lynparza, this review will highlight data of PARP inhibitors in in vitro and in vivo cancer models and explore some of the clinical applications and challenges of PARP inhibitor therapy.

\section{MECHANISMS OF ANTI-TUMOR EFFECT OF PARP INHIBITORS}

Poly (ADP-ribose) polymerase inhibitors are structurally similar in that they contain a nicotinamide moiety and mimic the $\mathrm{NAD}^{+}$substrate. PARP inhibitors competitively bind to the catalytic domain of PARPs and inhibit PAR synthesis with half-maximal inhibitory concentration $\left(\mathrm{IC}_{50}\right)$ values in 
TABLE 1 | PARP inhibitors in Phase 2 and Phase 3 clinical development ${ }^{\mathrm{a}}$.

\begin{tabular}{|c|c|c|c|}
\hline PARP inhibitor & Clinical trial & Therapy & Tumor type \\
\hline \multirow[t]{5}{*}{ Olaparib } & Phase 2 & Monotherapy & $\begin{array}{l}\text { Ovarian, peritoneal, fallopian tube, breast, colorectal, lung, Ewing's sarcoma, prostate, pancreatic, } \\
\text { advanced tumors }\end{array}$ \\
\hline & & Combination & Breast, ovarian, peritoneal, fallopian tube, endometrial, gastric, prostate, lung, pancreatic \\
\hline & Phase 3 & Monotherapy & Breast, ovarian \\
\hline & & Combination & Ovarian, peritoneal, fallopian tube, gastric \\
\hline & & Maintenance & Ovarian, pancreatic \\
\hline \multirow[t]{3}{*}{ Veliparib } & Phase 2 & Monotherapy & Ovarian, fallopian tube, peritoneal, breast \\
\hline & & Combination & $\begin{array}{l}\text { Breast, ovarian, peritoneal, fallopian tube, colorectal, lung, cervical, prostate, liver, glioblastoma, } \\
\text { melanoma, pancreatic, advanced tumors }\end{array}$ \\
\hline & Phase 3 & Combination & Breast, lung, glioblastoma \\
\hline \multirow[t]{3}{*}{ Rucaparib } & Phase 2 & Monotherapy & Breast, ovarian, fallopian, peritoneal, pancreatic \\
\hline & Phase 3 & Combination & Breast \\
\hline & & Maintenance & Ovarian, fallopian tube, peritoneal \\
\hline \multirow[t]{3}{*}{ Niraparib } & Phase 2 & Monotherapy & Ovarian \\
\hline & Phase 3 & Monotherapy & Breast \\
\hline & & Maintenance & Ovarian \\
\hline \multirow[t]{2}{*}{ Talazoparib } & Phase 2 & Monotherapy & Breast, ovarian, endometrial, advanced tumors \\
\hline & Phase 3 & Monotherapy & Breast \\
\hline E7016 & Phase 2 & Combination & Melanoma \\
\hline
\end{tabular}

${ }^{a}$ Completed and active clinical trials obtained from www. clinicaltrials. gov, data accessed August 2015.

the low nanomolar range (6-8). PARP inhibitors were developed to block the enzymatic activity of PARPs and prevent SSB repair by inhibiting the base excision repair (BER) pathway, and initial clinical development focused on potentiating the effects of chemotherapy and radiation $(6,9,10)$. Subsequent studies demonstrated that PARP inhibitors alone were cytotoxic in HR-deficient cells $(3,4,11)$. Based on these findings, a model was proposed in which PARP inhibition causes unrepaired SSBs, which are subsequently converted to DSBs, leading to synthetic lethality in HR-deficient cells (4). However, knockdown of XRCC1, the protein immediately downstream of PARP in the BER pathway did not lead to synthetic lethality (12), suggesting that loss of PARP activity is critical for synthetic lethality, but the loss of BER is not.

Poly (ADP-ribose) polymerases function in other aspects of DNA repair, and emerging data suggest other mechanisms of action for the anti-tumor activity of PARP inhibitors in HRdeficient cells $(13,14)$. One potential mechanism proposes that PARP inhibition activates NHEJ in HR-deficient cells, leading to genomic instability and cell death (12). In vitro studies have demonstrated that PARPs can regulate components of the NHEJ machinery, including DNA-dependent protein kinase (DNA-PK), $\mathrm{Ku} 70$, and Ku80 (15-18). In HR-deficient cells, PARP inhibitor treatment induced the activation of DNA-PK and phosphorylation of downstream substrates and increased NHEJ of a reporter plasmid containing a DSB (12). Pharmacological blockade or loss of NHEJ proteins reduced chromosomal aberrations and the cytotoxic effects of PARP inhibition, indicating a role for NHEJ in PARP inhibitor activity.

In vitro studies have demonstrated that the activity of PARP inhibitors may also involve formation of deleterious PARP-DNA complexes, which hinder DNA replication and repair (19-21).
Avian cells lacking PARP1 and PARP2 were resistant to olaparib treatment and remained viable at concentrations greater than $10 \mu \mathrm{M}$ (19). In contrast, olaparib caused significant cytotoxicity in wild type cells and increased levels of $\gamma$-H2AX, a marker of DNA damage. PAR polymers were undetectable by ELISA in both olaparib-treated wild type cells and PARP-deficient cells, suggesting that PARP inhibition is distinct from genetic deletion of PARP.

A comparison of PARP inhibitors demonstrated comparable inhibition of PAR synthesis by Western blot and ELISA (19, 20). In contrast, each PARP inhibitor showed varying ability to induce PARP-DNA complexes in the presence of alkylating agent. In the absence of PARP inhibitor, PARP1 was detected in the nuclear soluble fraction by Western blot and accumulated in the chromatin-bound fraction following PARP inhibitor treatment. In tumor cells, BMN 673 (talazoparib) induced greater accumulation of PARP1 and PARP2 in the chromatin-bound fraction compared to olaparib and rucaparib. Niraparib induced greater PARP-DNA binding than olaparib, and veliparib was the least effective enhancer of PARP-DNA binding at concentrations that maximally inhibited PARP enzymatic activity. PARP-DNA binding was detected at pharmacologically relevant concentrations and correlated with the cytotoxicity of each agent in vitro. In vivo, enhanced PARP-DNA binding did not correlate with better anti-tumor activity but resulted in increased toxicity (22). The significance of differential PARP-DNA binding on efficacy and tolerability requires further investigation in the context of different tumor types and different PARP inhibitor and chemotherapy regimens. The complex role of PARPs in cellular homeostasis, including DNA repair, highlights the need to evaluate PARP inhibitors for modulating other biological functions of PARPs. 


\section{FUTURE CHALLENGES OF PARP INHIBITOR DEVELOPMENT}

Clinical evaluation of the pharmacodynamic (PD) activity of PARP inhibitors has focused primarily on measuring inhibition of ex vivo enzymatic activity or PAR incorporation in tumor tissues and peripheral blood mononuclear cells (PBMCs). In a Phase 0 clinical trial, the National Cancer Institute and Abbott Laboratories validated a sandwich immunoassay to evaluate the PD response of veliparib during clinical development (23-25). The immunoassay measured changes in PARylated substrates collected from peripheral blood and tumor biopsy samples. While $\mathrm{PD}$ evaluations have demonstrated target engagement by veliparib and other PARP inhibitors, it is currently unclear what level of PARP inhibition is required to translate into a clinical response. In the case of olaparib, patients with BRCA-deficient ovarian or breast cancer demonstrated maximal PARP inhibition in PBMCs at doses greater than $60 \mathrm{mg}$ BID olaparib capsules; however, dosedependent anti-tumor activity was observed at higher doses of 100 and $400 \mathrm{mg}$ BID olaparib capsules (26-28).

Several factors may contribute to the lack of a clear relationship between PARP inhibition and clinical activity. Exploratory analysis of olaparib pharmacokinetic (PK)/PD data suggested that sustaining unbound steady-state trough concentrations above the $\mathrm{IC}_{90}$ for PARP inhibition affords better clinical efficacy. ${ }^{1}$ These results correlated with in vivo $\mathrm{PK} / \mathrm{PD}$ modeling of mouse tumor xenograft data that demonstrated a marked increase in DNA SSBs when PAR levels were decreased by more than $90 \%$, and exceeding this threshold improved the anti-tumor activity of olaparib in BRCA-deficient tumors. A simulation of unbound steady-state trough concentrations in patients receiving 100,200 , and $400 \mathrm{mg}$ BID olaparib capsules indicated that patients receiving $400 \mathrm{mg}$ BID achieved steady-state trough concentrations exceeding the $\mathrm{IC}_{90}$ value for PARP inhibition. Other potential reasons for lack of a PK/PD relationship include off-target effects of PARP inhibitors or variability in PK data. Another possibility is that the cytotoxicity of PARP inhibitors may involve other mechanisms of action.

To date, investigation of the mechanisms of resistance to PARP inhibitor anti-tumor effects has been limited $(29,30)$. Potential mechanisms of resistance to PARP inhibitors may involve restoration of HR or modulation of PARP itself. One potential mechanism was demonstrated in the Capan-1 human metastatic pancreatic adenocarcinoma cell line, which lacks a wild type copy of $B R C A$ while harboring a $6174 \mathrm{delT}$ mutant $B R C A$ allele. This mutation causes a frameshift in the normal open reading frame (ORF), resulting in expression of truncated BRCA protein and a deficiency in HR $(31,32)$. Analysis of Capan-1 clones resistant to PARP inhibitors showed that additional mutations (i.e., deletion, insertion, or deletion/insertion) within $B R C A$ in these cells rectified the 6174delT frameshift mutation and restored $B R C A 2$ normal ORF and BRCA function. Additional evidence that at least a partial restoration of HR can lead to resistance to

\footnotetext{
${ }^{1}$ Pharmacology/Toxicology NDA review: olaparib 2014. Available from: http: //www.accessdata.fda.gov/drugsatfda_docs/nda/2014/206162Orig1s000PharmR. pdf
}

PARP inhibitors include secondary mutations in the BRCA gene, restoring expression of wild type BRCA protein in patients (33) and somatic mutation of TP53BP1 $(34,35)$.

In addition to restoration of HR, studies have also correlated resistance to PARP inhibitors with PARP itself and PD markers such as $\gamma$-H2AX $(36,37)$. In an in vivo study, responsiveness of mice bearing TC-71 Ewing sarcoma tumors to a combination of talazoparib and temozolamide was correlated with decreased levels of total or cleaved PARP and increases in $\gamma-\mathrm{H} 2 \mathrm{AX}$; however, tumors that were resistant to the combination treatment were shown to have some cleaved PARP but no decrease in total or cleaved PARP, or increases in $\gamma-\mathrm{H} 2 \mathrm{AX}$ (38). Although the status of the genes involved in HR was not evaluated in tumors tested in this study, these results suggest that another potential mechanism of resistance to anti-tumor effects of PARP inhibitors may involve regulation of PARP itself.

The most concerning potential adverse reactions associated with PARP inhibition are myelodysplastic syndrome and acute myeloid leukemia (MDS/AML), especially in patients harboring a germline BRCA mutation. BRCA1 is critically involved with the Fanconi anemia proteins in repairing DNA damage, whereas BRCA2 is itself a Fanconi anemia protein. Biallelic mutations of $B R C A 2$ are linked to Fanconi's anemia, a genetic disorder characterized by congenital abnormalities and a profound increase in cancer predisposition, namely AML $(39,40)$. The U.S. Package Insert for Lynparza (olaparib) contains the following warning for the development of MDS/AML: MDS/AML have been confirmed in 6 out of 298 (2\%) patients enrolled in a single arm trial of Lynparza monotherapy, in patients with deleterious or suspected deleterious germline BRCA-mutated advanced cancers $^{2}$. In a randomized placebo controlled trial, MDS/AML occurred in 3 out of $136(2 \%)$ patients with advanced ovarian cancer treated with Lynparza. Overall, MDS/AML were reported in 22 of 2,618 $(<1 \%)$ patients treated with Lynparza. The majority of MDS/AML cases (17 of 22 cases) were fatal, and the duration of therapy with Lynparza in patients who developed secondary MDS/cancer-therapy related AML varied from $<6$ months to $>2$ years. All patients had previous chemotherapy with platinum agents and/or other DNA damaging agents. The addition of further DNA damage induced by chemotherapy or other environmental factors, coupled with enhanced impairment of a compensatory repair pathway by means of PARP inhibition, may prime patients with germline DNA repair deficiencies for the development of MDS/AML. Monitoring of complete blood counts and perhaps PBMCs for micronuclei is warranted for patients receiving PARP inhibitors, and further investigations should be performed for prolonged hematologic toxicity (see text footnote 2).

\section{CONCLUSION}

Notwithstanding the current knowledge regarding the biological role of PARP and its demonstrated clinical benefit in cancers with germline $B R C A$ mutations, future studies are needed to improve

${ }^{2}$ LYNPARZA $^{\text {sw }}$ (olaparib) label: Available from http://www.accessdata.fda.gov/ scripts/cder/drugsatfda/ 
the therapeutic potential of PARP inhibitors. For example, better understanding of the contribution of the various mechanisms of action in vivo, in the context of different PARP inhibitors and different tumor types, together with better understanding of mechanisms of resistance will aid in improving the therapeutic potential of this class of drugs by optimizing patient selection (e.g., based on baseline or PARP inhibitor-mediated changes in HRD profile) or optimizing selection of therapeutic agents in combination clinical trials by targeting separate mechanisms of drug

\section{REFERENCES}

1. Gibson BA, Kraus WL. New insights into the molecular and cellular functions of poly(ADP-ribose) and PARPs. Nat Rev Mol Cell Biol (2012) 13:411-24. doi:10.1038/nrm3376

2. Schreiber V, Dantzer F, Ame JC, de Murcia G. Poly(ADP-ribose): novel functions for an old molecule. Nat Rev Mol Cell Biol (2006) 7:517-28. doi:10.1038/ nrm 1963

3. Bryant HE, Schultz N, Thomas HD, Parker KM, Flower D, Lopez E, et al. Specific killing of BRCA2-deficient tumours with inhibitors of poly(ADP-ribose) polymerase. Nature (2005) 434:913-7. doi:10.1038/nature03443

4. Farmer H, McCabe N, Lord CJ, Tutt AN, Johnson DA, Richardson TB, et al. Targeting the DNA repair defect in BRCA mutant cells as a therapeutic strategy. Nature (2005) 434:917-21. doi:10.1038/nature03445

5. Kim G, Ison G, McKee AE, Zhang H, Tang S, Gwise $T$, et al. FDA approval summary: olaparib monotherapy in patients with deleterious germline BRCA-mutated advanced ovarian cancer treated with three or more lines of chemotherapy. Clin Cancer Res (2015) 21:4257-61. doi:10.1158/1078-0432. CCR-15-0887

6. Curtin NJ, Szabo C. Therapeutic applications of PARP inhibitors: anticancer therapy and beyond. Mol Aspects Med (2013) 34:1217-56. doi:10.1016/j.mam. 2013.01.006

7. Rouleau M, Patel A, Hendzel MJ, Kaufmann SH, Poirier GG. PARP inhibition: PARP1 and beyond. Nat Rev Cancer (2010) 10:293-301. doi:10.1038/nrc2812

8. Shen Y, Rehman FL, Feng Y, Boshuizen J, Bajrami I, Elliott R, et al. BMN 673, a novel and highly potent PARP1/2 inhibitor for the treatment of human cancers with DNA repair deficiency. Clin Cancer Res (2013) 19:5003-15. doi:10.1158/ 1078-0432.CCR-13-1391

9. Plummer R, Jones C, Middleton M, Wilson R, Evans J, Olsen A, et al. Phase I study of the poly(ADP-ribose) polymerase inhibitor, AG014699, in combination with temozolomide in patients with advanced solid tumors. Clin Cancer Res (2008) 14:7917-23. doi:10.1158/1078-0432.CCR-08-1223

10. Durkacz BW, Omidiji O, Gray DA, Shall S. (ADP-ribose)n participates in DNA excision repair. Nature (1980) 283:593-6. doi:10.1038/283593a0

11. McCabe N, Turner NC, Lord CJ, Kluzek K, Bialkowska A, Swift S, et al. Deficiency in the repair of DNA damage by homologous recombination and sensitivity to poly(ADP-ribose) polymerase inhibition. Cancer Res (2006) 66:8109-15. doi:10.1158/0008-5472.CAN-06-0140

12. Patel AG, Sarkaria JN, Kaufmann SH. Nonhomologous end joining drives poly(ADP-ribose) polymerase (PARP) inhibitor lethality in homologous recombination-deficient cells. Proc Natl Acad Sci U S A (2011) 108:3406-11. doi:10.1073/pnas.1013715108

13. De Lorenzo SB, Patel AG, Hurley RM, Kaufmann SH. The elephant and the blind men: making sense of PARP inhibitors in homologous recombination deficient tumor cells. Front Oncol (2013) 3:228. doi:10.3389/fonc.2013.00228

14. Helleday T. The underlying mechanism for the PARP and BRCA synthetic lethality: clearing up the misunderstandings. Mol Oncol (2011) 5:387-93. doi: 10.1016/j.molonc.2011.07.001

15. Galande S, Kohwi-Shigematsu T. Poly(ADP-ribose) polymerase and $\mathrm{Ku}$ autoantigen form a complex and synergistically bind to matrix attachment sequences. J Biol Chem (1999) 274:20521-8. doi:10.1074/jbc.274.29.20521

16. Li B, Navarro S, Kasahara N, Comai L. Identification and biochemical characterization of a Werner's syndrome protein complex with Ku70/80 and poly(ADP-ribose) polymerase-1. J Biol Chem (2004) 279:13659-67. doi:10. 1074/jbc.M311606200 resistance. Additionally, studies are needed to identify predictive biomarkers and to develop validated, diagnostic tests to extend the therapeutic landscape of PARP inhibitors beyond BRCA-mutated tumors (41-43).

\section{AUTHOR NOTE}

This article reflects the views of the authors and should not be construed to represent FDA's views and policies.

17. Pleschke JM, Kleczkowska HE, Strohm M, Althaus FR. Poly(ADP-ribose) binds to specific domains in DNA damage checkpoint proteins. J Biol Chem (2000) 275:40974-80. doi:10.1074/jbc.M006520200

18. Wang M, Wu W, Wu W, Rosidi B, Zhang L, Wang H, et al. PARP-1 and Ku compete for repair of DNA double strand breaks by distinct NHEJ pathways. Nucleic Acids Res (2006) 34:6170-82. doi:10.1093/nar/gkl840

19. Murai J, Huang SY, Das BB, Renaud A, Zhang Y, Doroshow JH, et al. Trapping of PARP1 and PARP2 by clinical PARP Inhibitors. Cancer Res (2012) 72:5588-99. doi:10.1158/0008-5472.CAN-12-2753

20. Murai J, Huang SY, Renaud A, Zhang Y, Ji J, Takeda S, et al. Stereospecific PARP trapping by BMN 673 and comparison with olaparib and rucaparib. Mol Cancer Ther (2014) 13:433-43. doi:10.1158/1535-7163.MCT-13-0803

21. Strom CE, Johansson F, Uhlen M, Szigyarto CA, Erixon K, Helleday T. Poly (ADP-ribose) polymerase (PARP) is not involved in base excision repair but PARP inhibition traps a single-strand intermediate. Nucleic Acids Res (2011) 39:3166-75. doi:10.1093/nar/gkq1241

22. Hopkins TA, Shi Y, Rodriguez LE, Solomon LR, Donawho CK, Digiammarino EL, et al. Mechanistic dissection of PARP1 trapping and the impact on in vivo tolerability and efficacy of PARP Inhibitors. Mol Cancer Res (2015). doi:10.1158/ 1541-7786.MCR-15-0191-T

23. Kinders RJ, Hollingshead M, Khin S, Rubinstein L, Tomaszewski JE, Doroshow $\mathrm{JH}$, et al. Preclinical modeling of a phase 0 clinical trial: qualification of a pharmacodynamic assay of poly (ADP-ribose) polymerase in tumor biopsies of mouse xenografts. Clin Cancer Res (2008) 14:6877-85. doi:10.1158/1078-0432. CCR-08-0214

24. Kummar S, Kinders R, Gutierrez ME, Rubinstein L, Parchment RE, Phillips LR, et al. Phase 0 clinical trial of the poly (ADP-ribose) polymerase inhibitor ABT888 in patients with advanced malignancies. J Clin Oncol (2009) 27:2705-11. doi:10.1200/JCO.2008.19.7681

25. Ji J, Kinders RJ, Zhang Y, Rubinstein L, Kummar S, Parchment RE, et al. Modeling pharmacodynamic response to the poly(ADP-Ribose) polymerase inhibitor ABT-888 in human peripheral blood mononuclear cells. PLoS One (2011) 6:e26152. doi:10.1371/journal.pone.0026152

26. Audeh MW, Carmichael J, Penson RT, Friedlander M, Powell B, Bell-McGuinn $\mathrm{KM}$, et al. Oral poly(ADP-ribose) polymerase inhibitor olaparib in patients with BRCA1 or BRCA2 mutations and recurrent ovarian cancer: a proof-of-concept trial. Lancet (2010) 376:245-51. doi:10.1016/S0140-6736(10)60893-8

27. Tutt A, Robson M, Garber JE, Domchek SM, Audeh MW, Weitzel JN, et al. Oral poly(ADP-ribose) polymerase inhibitor olaparib in patients with BRCA1 or BRCA2 mutations and advanced breast cancer: a proof-of-concept trial. Lancet (2010) 376:235-44. doi:10.1016/S0140-6736(10)60892-6

28. Fong PC, Boss DS, Yap TA, Tutt A, Wu P, Mergui-Roelvink M, et al. Inhibition of poly(ADP-ribose) polymerase in tumors from BRCA mutation carriers. $N$ Engl J Med (2009) 361:123-34. doi:10.1056/NEJMoa0900212

29. Bouwman P, Jonkers J. Molecular pathways: how can BRCA-mutated tumors become resistant to PARP inhibitors? Clin Cancer Res (2014) 20:540-7. doi:10. 1158/1078-0432.CCR-13-0225

30. Lord CJ, Ashworth A. Targeted therapy for cancer using PARP inhibitors. Curr Opin Pharmacol (2008) 8:363-9. doi:10.1016/j.coph.2008.06.016

31. Edwards SL, Brough R, Lord CJ, Natrajan R, Vatcheva R, Levine DA, et al. Resistance to therapy caused by intragenic deletion in BRCA2. Nature (2008) 451:1111-5. doi:10.1038/nature06548

32. Sakai W, Swisher EM, Karlan BY, Agarwal MK, Higgins J, Friedman C, et al Secondary mutations as a mechanism of cisplatin resistance in BRCA2-mutated cancers. Nature (2008) 451:1116-20. doi:10.1038/nature06633 
33. Barber LJ, Sandhu S, Chen L, Campbell J, Kozarewa I, Fenwick K, et al. Secondary mutations in BRCA2 associated with clinical resistance to a PARP inhibitor. J Pathol (2013) 229:422-9. doi:10.1002/path.4140

34. Fojo T, Bates S. Mechanisms of resistance to PARP inhibitors three and counting. Cancer Discov (2013) 3:20-3. doi:10.1158/2159-8290. CD- $12-0514$

35. Jaspers JE, Kersbergen A, Boon U, Sol W, van Deemter L, Zander SA, et al. Loss of 53BP1 causes PARP inhibitor resistance in Brcal-mutated mouse mammary tumors. Cancer Discov (2013) 3:68-81. doi:10.1158/2159-8290.CD12-0049

36. Lowndes NF, Toh GW. DNA repair: the importance of phosphorylating histone H2AX. Curr Biol (2005) 15:R99-102. doi:10.1016/j.cub.2005.01.029

37. Rogakou EP, Pilch DR, Orr AH, Ivanova VS, Bonner WM. DNA doublestranded breaks induce histone H2AX phosphorylation on serine 139. J Biol Chem (1998) 273:5858-68. doi:10.1074/jbc.273.10.5858

38. Smith MA, Reynolds CP, Kang MH, Kolb EA, Gorlick R, Carol H, et al. Synergistic activity of PARP inhibition by talazoparib (BMN 673) with temozolomide in pediatric cancer models in the pediatric preclinical testing program. Clin Cancer Res (2015) 21:819-32. doi:10.1158/1078-0432.CCR-14-2572

39. D'Andrea AD. Susceptibility pathways in Fanconi's anemia and breast cancer. $N$ Engl J Med (2010) 362:1909-19. doi:10.1056/NEJMra0809889

40. Meyer S, Tischkowitz M, Chandler K, Gillespie A, Birch JM, Evans DG. Fanconi anaemia, BRCA2 mutations and childhood cancer: a developmental perspective from clinical and epidemiological observations with implications for genetic counselling. J Med Genet (2014) 51:71-5. doi:10.1136/jmedgenet-2013-101642

41. McNeish IA, Oza AM, Coleman RL, Scott CL, Konecny GE, Tinker A, et al. Results of ARIEL2: a Phase 2 trial to prospectively identify ovarian cancer patients likely to respond to rucaparib using tumor genetic analysis. ASCO Meeting Abstracts (2015) 33:5508.

42. Watkins JA, Irshad S, Grigoriadis A, Tutt AN. Genomic scars as biomarkers of homologous recombination deficiency and drug response in breast and ovarian cancers. Breast Cancer Res (2014) 16:211. doi:10.1186/bcr3670

43. Michels J, Vitale I, Saparbaev M, Castedo M, Kroemer G. Predictive biomarkers for cancer therapy with PARP inhibitors. Oncogene (2014) 33:3894-907. doi:10. 1038/onc.2013.352

Conflict of Interest Statement: The authors declare that the research was conducted in the absence of any commercial or financial relationships that could be construed as a potential conflict of interest.

Copyright (C) 2015 Ricks, Chiu, Ison, Kim, McKee, Kluetz and Pazdur. This is an openaccess article distributed under the terms of the Creative Commons Attribution License (CC BY). The use, distribution or reproduction in other forums is permitted, provided the original author(s) or licensor are credited and that the original publication in this journal is cited, in accordance with accepted academic practice. No use, distribution or reproduction is permitted which does not comply with these terms. 